\title{
In situ Monitoring of Pavement Stresses on the A1 in Switzerland
}

\begin{abstract}
A large number of sensors installed as part of the European cooperative project, Eureka Logchain Footprint, are used to examine the effects of individual vehicles on the road pavement and the environment. The weigh-in-motion data recorded at the Footprint Monitoring Site near Lenzburg, Switzerland are analyzed and figures are presented which describe the nature of the traffic experienced at the monitoring site. The weighin-motion data are matched to data from a prototype stress-in-motion sensor from the same site and the two datasets are compared. The ability of the stress-in-motion sensor to record the contact stress distribution is discussed and procedures are proposed for describing the shape of the distribution using a relatively small number of parameters. A method for quantifying distribution shape in terms of an "m"-ness value is proposed. A finite element model of the road is constructed, validated, and used to predict the stresses and strains in the pavement related to particular contact stress distributions. It is demonstrated that, for all but the heaviest tires, the shape of the stress distribution has a significant effect on the stresses and strains within the pavement.
\end{abstract}

KEYWORDS: stress-in-motion, weigh-in-motion, in situ monitoring, finite element modeling

\section{Introduction}

\section{Previous Studies}

The accurate measurement of the stress distribution under the tires of moving vehicles is important for the understanding of pavement behavior and the modeling of pavement failure. Previous studies have shown that the contact stress distribution under tires is highly nonuniform and can produce localized areas of very high stress and strain on the pavement surface [1-3]. Other studies have focused on the direct measurement of strains in trafficked pavements [4-6]. It is not only important to discover an accurate method of relating the contact stresses to pavement performance, but also to find a way to predict which vehicles, axles, and tires will produce the highest stresses and strains.

Many of these previous studies have focused on the measurement of stress distributions of known tires under laboratory conditions $[2,3,5]$, relating factors such as tire pressure, axle load, and tire construction to the stress distribution and hence to pavement damage. It is also important, however, to find a method for predicting which axles are more damaging to the road pavement in situ (and without removing vehicles from the traffic stream as in Ref. [1]), where the tires' pressures and models and the vehicle's characteristics are unknown and uncontrolled.

\section{Project Overview}

Since June 2005, an in situ measuring station has been in operation on the A1 motorway in Lenzburg, Switzerland between Zürich and Bern. The Footprint Monitoring Station (FMS) is part of the ongoing European cooperative project, Eureka Logchain

Manuscript received August 17, 2007; accepted for publication March 10, 2008; published online April 2008.

${ }^{1}$ Empa Swiss Federal Laboratories for Materials Testing and Research, Ueberlandstrasse 129, Duebendorf, ZH 8600, Switzerland, e-mail: lily.poulikakos@empa.ch
Footprint [7,12], and was constructed in tandem with a weigh-inmotion (WIM) site. The data collected from this site is used in this paper to give an overview of the type and density of traffic on the A1 and the tire contact stresses experienced by the pavement. The data are analyzed in an attempt to relate parameters which can be easily measured in situ to the damage done to the road by particular vehicles and axles.

\section{Sensor Installation}

\section{The Footprint Measuring Station}

The FMS, on completion, consisted of a large number of sensors installed in or near the road pavement of the slow (outermost) lane of the A1. A schematic of the site is provided in Fig. 1. The sensors relevant to this paper are as follows.

\section{The Weigh-in-Motion Sensors}

Two pairs of Lineas ${ }^{\mathrm{TM}}$ WIM sensors [9] were installed in the pavement by the Swiss Federal Roads Office (FERDRO/ ASTRA). These sensors give the following parameters for each vehicle that crosses them with a gross vehicle weight (GVW) of over three metric tons:

- Axle load (for each axle of the vehicle)

- Speed

- Distances between axles

- Distances between vehicles

- "Swiss 10" classification

\section{The Stress-in-Motion Sensors}

Two Modulas ${ }^{\mathrm{TM}}$ SIM sensor arrays (Fig. 1 and 2) were installed in the road. These sensors, like the WIM sensors on the same site, are quartz-based piezoelectric sensors. Quartz-based sensors have shown unique qualities as they are electrically and mechanically 


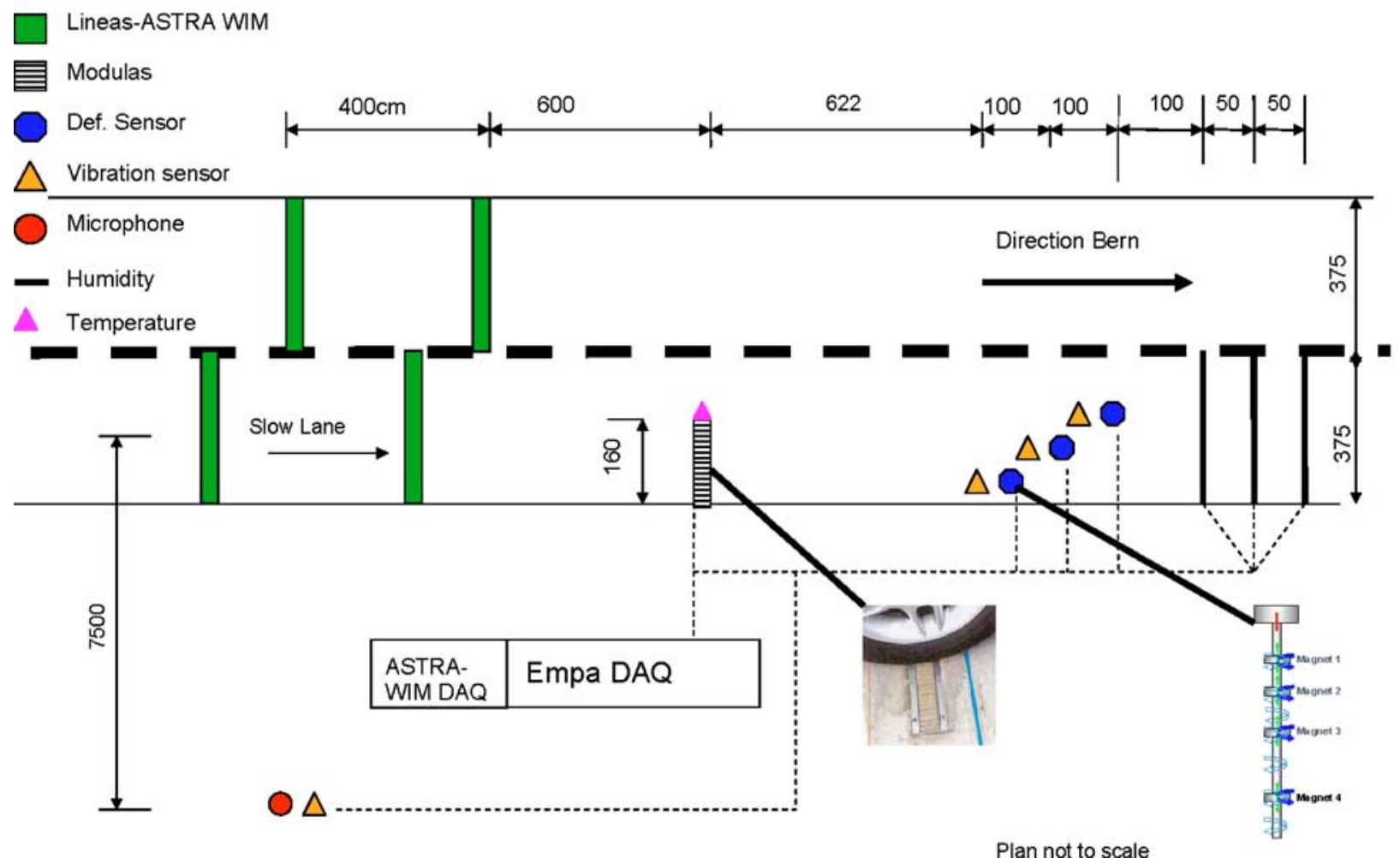

FIG. 1-Schematic of the Footprint Monitoring Station.

stable, temperature influences are negligible, show uniform sensitivity over the entire sensor, display a wide measurement range, and results are speed independent [8]. Additionally, during prior laboratory tests it was shown that the effect of speed on the results of the SIM sensor is minimal. These results are published elsewhere.

Each sensor array consists of 32 separate piezoelectric sensors, each 50 by $14.5 \mathrm{~mm}$. The array is capable of recording the force distribution on each one of these sensors at a frequency of $16392 \mathrm{~Hz}$ [8]. In this installation, two SIM sensor arrays were fitted side-by-side, giving 64 sensors (or channels) arranged in a line across the right-hand wheel path.

The sensors were fitted into a precast concrete block. This en-

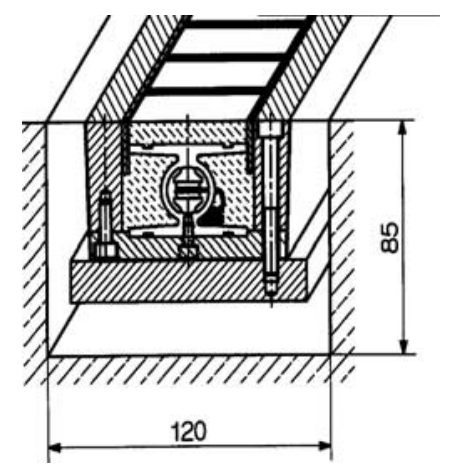

FIG. 2-Section through one SIM sensor array (dimensions in mm). [8] sured that the sensor had a solid foundation and that the deflection and movement of the sensor under loading would be minimal, ensuring good quality measurements and prolonging the life of the sensor. As the installation of this sensor was performed overnight, with limited visibility and time, the concrete block allowed the installation to be simpler and more precise. It also permitted the sensor to be removed temporarily for maintenance or inspection without major works or lane closures on the motorway.

The installed SIM sensor gives the contact stress distribution under each half axle that crosses it. An example of the output is shown in Fig. 3. Either force or stress distributions may be plotted, as the area of each sensor element is constant $\left(750 \mathrm{~mm}^{2}\right)$. Only events in which one or more of the channels registers over $1000 \mathrm{~N}$ are recorded.

\section{Temperature Sensors}

Two groups of temperature sensors were installed alongside the SIM sensor in the pavement. Each group consisted of three separate sensors, positioned at the boundaries between asphalt layers in the road construction as shown in Fig. 4. Each sensor provided the temperature of the asphalt at each depth once every minute.

\section{Acceleration Sensors}

Overnight on the 30th of June 2005, three acceleration sensors were installed in the road and were trafficked by two vehicles of 


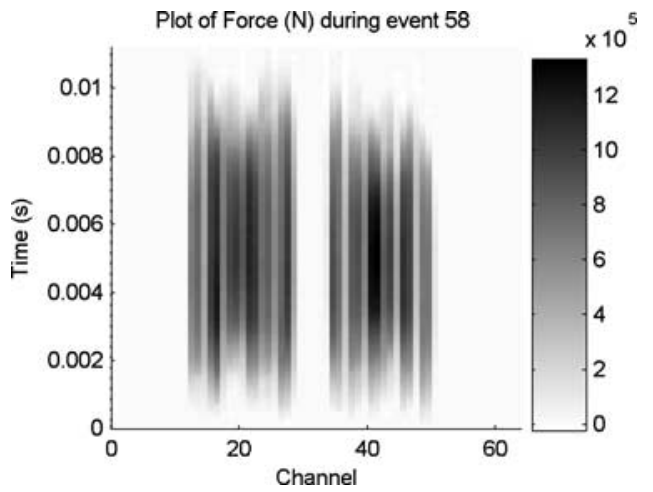

FIG. 3-SIM sensor results from a dual tire with " $n$ "-shaped stress distributions.

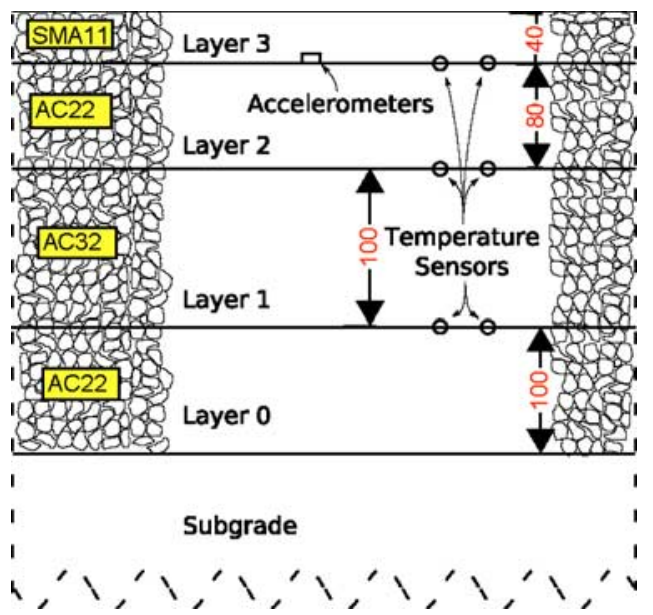

FIG. 4-Schematic of the A1 pavement showing layer numbering, type of material, and depths of temperature and acceleration sensors (dimensions in $\mathrm{mm}$ ).

known loading and dimensions. The sensors were fitted at the base of pavement Layer 3 as shown in Fig. 4 and recorded the vertical acceleration of the ground at a frequency of $255 \mathrm{~Hz}$. Postprocessing the recorded waveforms describing the acceleration during a vehicle pass with a numerical integration gave an estimate of the road surface deflection using a method described by Arraigada [10].

\section{Recorded Data}

\section{Analysis of WIM Data}

Data on passing vehicles and axles has been recorded almost continuously since June 2005. The data upon which this study focuses are taken from September 2005.

The WIM recorded 90498 vehicles with a combined total of 283113 axles during September 2005. For one sample day of September, Fig. 5 shows the distribution of these vehicles according to the "Swiss 10" vehicle classification. This system divides vehicles into ten categories as shown in Table 1 . As only the records of vehicles with gross weights over 3 tons are stored, there are a relatively small number of Class 3 (car) events compared to the Class 10 (articulated trucks).

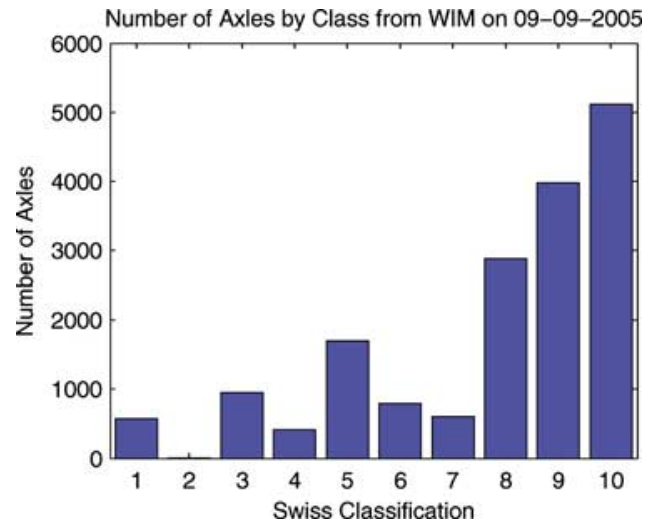

FIG. 5-Histogram of axles on the A1 according to the "Swiss 10" classification.

TABLE 1-The "Swiss 10" vehicle classification system.

\begin{tabular}{lcc}
\hline Class & Vehicle Type & Axles \\
\hline 1 & Buses and Coaches & $2-5$ \\
2 & Motorcycles & 2 \\
3 & Personal Cars & 2 \\
4 & Personal Cars with Trailers & $3-4$ \\
5 & Delivery/Pick-up Trucks $(<3.5$ t $)$ & 2 \\
6 & Delivery/Pick-up Trucks with Trailers $(<3.5$ t $)$ & 3 \\
7 & Articulated Delivery/Pick-up Trucks with Semi-Trailers & 3 \\
8 & Freight Trucks & $2-4$ \\
9 & Freight Trucks with Trailers & $3-6$ \\
10 & Articulated Freight Trucks with Semi-Trailers & $>3$ \\
\hline
\end{tabular}

Figure 6 shows how the axle loads are distributed among the classes of the "Swiss 10" using a box plot. This shows the median and upper and lower quartiles, as well as highlighting outliers [11]. It can be seen that the boxes for all the classes fall well below 10 tons, indicating that the vast majority of the axles are unlikely to infringe upon Swiss regulations on axle load. ${ }^{2}$

Figure 7 shows the distribution of vehicles (as opposed to the axles in Fig. 5) and highlights what proportion of them can be shown to infringe upon the Swiss regulations on axle load using the WIM data. The WIM sensor is unable to detect the type of suspension or date of vehicle registration, resulting in the portion of vehicles which might infringe upon the regulations. Using this finer classification reduces the number of axles identified as overloaded.

It can be seen from Figs. 5-7 that the proportion of vehicles which infringe upon existing axle load regulations is small but not insignificant. The results from the SIM sensor were used to more closely examine some of these vehicles with high axle loads.

\section{Analysis of SIM Data}

Post-Processing and Visualization - The data recorded by the SIM sensor can be considered in two ways. The first, and simplest, is to simply convert the recorded force, $P z(t)$, from the SIM sensor into stress by dividing by the sensor area $\left(750 \mathrm{~mm}^{2}\right)$. This method is simple and quick, and produces results like those

${ }^{2}$ This is a coarse approximation to the regulations, which also takes into account group-weights, suspension, and date of vehicle registration. 


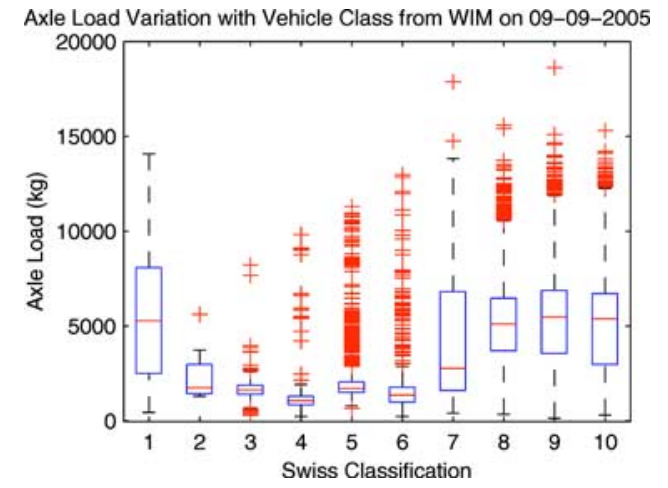

FIG. 6-Box-plot of axle load on the A1 grouped according to the "Swiss 10" classification.

presented in Fig. 3, with a relatively erratic distribution normal to the direction of travel, and a smooth, bell-shaped curve parallel to the direction of travel. This smooth curve is due to the fact that, in general, motorway vehicles do not move at a speed such that each force recorded by the sensor is discrete. For example, it takes 2 $\times 10^{-3} \mathrm{~s}$ for a vehicle at the speed limit to travel $5 \mathrm{~cm}$ (the length of the Modulas sensor), but the sensor in the road makes one record every $1.2 \times 10^{-4} \mathrm{~s}$. The effect of this is that the force/stress distribution that is recorded is smoothed. The SIM sensor essentially records the moving average of the force distribution. Alternatively, we can post-process the data recorded by the SIM sensor in an attempt to undo the effects of this smoothing. By considering the recorded forces from the surrounding records, the recorded force, $P z(t)$, can be converted into the unsmoothed force, $F_{z}$, using Eq. 1.

$$
F_{z}(t+\delta t)=P_{z}(t+\delta t)-\left[P_{z}(t)-F_{z}\left(t-\frac{L_{s}}{u_{x}}\right)\right]
$$

where $L_{s}$ is the sensor length $(5 \mathrm{~cm}), t$ is the time, $\delta t$ is the time interval between records and $u_{x}$ is the vehicle's speed.

While this theoretically produces much more detailed results, there are several practical problems associated with its use. In order to reduce the data storage and post-processing requirements, data from the SIM sensor were only stored when one or more channels was recording a force in excess of $1000 \mathrm{~N}$. The result of this is that Eq. 1 cannot be applied accurately to the first part of the event (from $t=0$ to $t=L_{s} / u_{x}$ ), which introduces a periodic error into the postprocessed results.

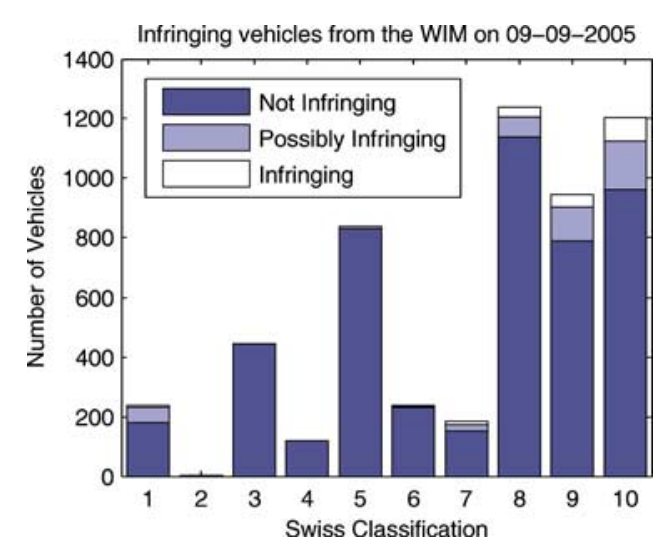

FIG. 7-Histogram of vehicles on the A1 grouped according to the "Swiss 10" classification with the overweight vehicles highlighted.
Wheel Load - The amount of SIM data for any given event is significantly larger than the amount of WIM data and, for most purposes, some method of data reduction is therefore needed. The wheel load could be directly estimated from the recorded forces, $P_{z}$. The method adopted was to effectively scale the recorded force measurements by the ratio of the timestep to the pass-by time $\left(L_{s} / u_{x}\right)$ for each channel. This must be discretized and summed for all the SIM sensor channels to give Eq. 2:

$$
F_{z t o t}=\delta t \times \frac{u_{x}}{L_{s}} \times \sum_{a}^{b} \sum_{n=0}^{n=N} P_{z}(n \times \delta t)
$$

where $F_{z t o t}$ is the total wheel load, $P z(t)$ is the force on a channel sensor at time $t, a \rightarrow b$ defines all the active channels and $n$ is the number of time steps from the start of the event. Other symbols are the same as Eq. 1.

The data from September 2005 were used to verify this equation for real, in situ vehicles, as previously it had only been applied under laboratory conditions with simulated loads. Figure 8 is a plot of $F_{z t o t}$ for events during four days from September against the halves of axle loads recorded by the WIM for the same events. ${ }^{3}$ There is a strong correlation along the line $F_{z t o t}=1.3 \times($ axle load $) / 2$, with $76 \%$ of the SIM-calculated wheel loads from these four days falling within $\pm 20 \%$ of this. It is clear from the figure, however, that there are a very significant number of outliers, necessitating the use of a robust regression algorithm. These outliers are thought to be due to inaccuracies in the matching of WIM and SIM results. Currently this inaccuracy is being corrected by implementing a unified data recording system.

Distribution Shape-Contact stress distributions can be classified as either " $n$ " or " $m$ "-shaped and the distributions from the road also follow this pattern, where " $n$ " refers to a singlepeaked distribution and " $m$ " refers to a double-peaked distribution [1]. Previous studies have tended to simply examine the distributions and visually judge whether the distribution is " $m$ " or " $n$ "shaped. For this project, due to the large amount of data potentially available, it was clear that an automatable method for describing the shape of the stress distribution was required. The following method for quantifying the shape of the distribution under a single tire is therefore proposed, which classifies the tire shape using two values, the "m"-ness and the skew, where the skew gives the extent to which the distribution is skewed to the left or right. The method is also represented graphically in Fig. 9.

1. Perform a moving average smoothing between adjacent channels such that the force on any channel, $i$, as a function of time is: Perform a moving average smoothing between each group of five adjacent channels such that the force, $F$, on the central channel of the five, $i$, as a function of time is:

$$
F_{i}(t)=\frac{1}{9}\left(F_{i-2}(t)+2 F_{i-1}(t)+3 F_{i}(t)+2 F_{i+1}(t)+F_{i+2}(t)\right)
$$

2. Perform a cumulative sum of the force functions for each channel, scaling between 0 and 1 , giving the cumulative force function, $C_{i}(t)$ :

\footnotetext{
${ }^{3}$ This assumes that both wheels of an axle are balanced.
} 


\section{Plot of recorded WIM loads with MODULAS calculations (Method F2)}

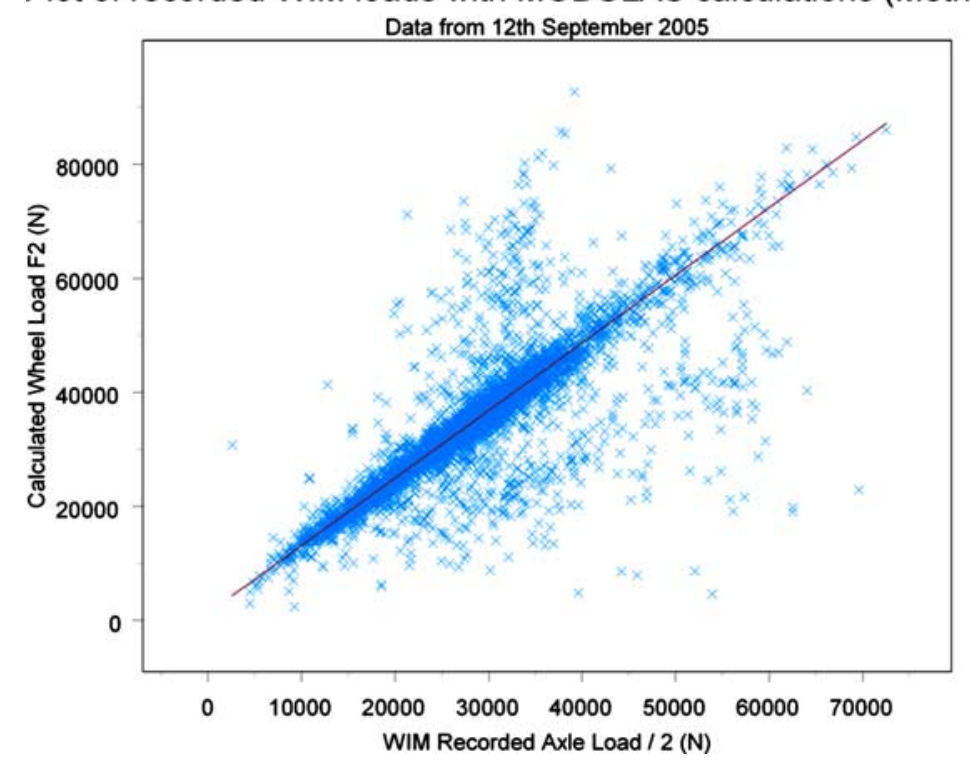

FIG. 8-Scatter graph showing the SIM calculated wheel load against the WIM calculated wheel load (both in N).

$$
C_{i}(t)=\frac{\sum_{n=1}^{n=i} F_{n}(t)}{\sum_{n=1}^{n=N} F_{n}(t)}
$$

where $N$ is the number of active channels.

3. Time-average the resulting cumulative force function, $C_{i}(t)$ to give the characteristic cumulative function, $C_{i}$ :

$$
C_{i}=\frac{\int_{t_{0}}^{t_{\text {end }}} C_{i}(t) d t}{t_{\text {end }}-t_{0}}
$$

4. The "m"-ness, $m$, is then given by:

$$
m=\sum_{n=1}^{n=\frac{N}{2}}\left[C_{n}-\frac{n}{N}\right]-\sum_{n=\frac{N}{2}}^{n=N}\left[C_{n}-\frac{n}{N}\right]
$$

and the skew, $s$, is given by:

$$
S=\sum_{n=1}^{n=\frac{N}{2}}\left[C_{n}-\frac{n}{N}\right]+\sum_{n=\frac{N}{2}}^{n=N}\left[C_{n}-\frac{n}{N}\right]
$$

It was discovered that this method produced results which were comparable with the classifications which had previously been assigned to tires purely by the visual inspection method. From a random sample of 100 wheels (with 122 tires), which had been classified both visually and using the method outlined above. The two methods agreed on 105 of the tires, disagreed on 16 of the tires, and one tire was not classifiable using the "m"-ness method.

\section{Some Sample Results}

Figure 3 has already illustrated an example of a dual, "n"-shaped distribution. Figure 10 gives an example of a single, "m"-shaped distribution. It is clear from the figure that the areas of high stress (dark) are located at the edges of the distribution, while the center of the distribution is generally lighter in color, indicating lower contact stresses. Conversely, the "n"-shaped tires shown in Fig. 3 tended to be darker (high stress) in the center and lighter (low stress) at the edges. Figure 11 shows an exceptional distribution recorded on the A1: the right-hand tire is severely overloaded (approximately $83.9 \mathrm{kN}$ ), while the inner, left-hand tire appears to be flat, and carries hardly any of the load. Figure 12 shows a threedimensional representation of a dual, "m"-shaped tire with a significant overload. The tire in Fig. 12 is carrying $11.5 \mathrm{kN}$ more load than the tire in Fig. 11.

\section{Stresses and Strains in the Road}

Finite Element Model-An explicit, dynamic finite element model of the road was developed using the commercial ABAQUS [13] code with the following key features:

- Three-dimensional geometry.

- Visco-elastic material model based on Prony series for each layer.

- Multi-layered, with the layers' geometry and material properties having been determined by lab measurements on asphalt cores taken from the road during sensor installation.

- Element spacing which gave a one-to-one correspondence between model elements and the SIM sensor elements.

- Layer boundaries which can either be fixed or use a defined coefficient of friction.

Validation - Currently, the finite element model was validated using the events with known vehicles and for which acceleration data were recorded on 30th June 2005. Two vehicles trafficked the sensor (and the entire FMS) repeatedly, at various speeds. The two cases that were considered for model validation were as follows:

- Vehicle 6102: two axles of $2350 \mathrm{~kg}$ and $5550 \mathrm{~kg}$, respectively, traveling at $50 \mathrm{~km} / \mathrm{h}$.

- Vehicle 6103: three axles of $4200 \mathrm{~kg}, 4500 \mathrm{~kg}$, and $2550 \mathrm{~kg}$, 
SIM sensor channels

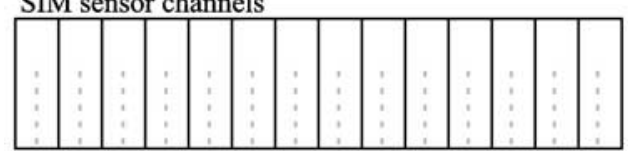

'm'-shaped tire
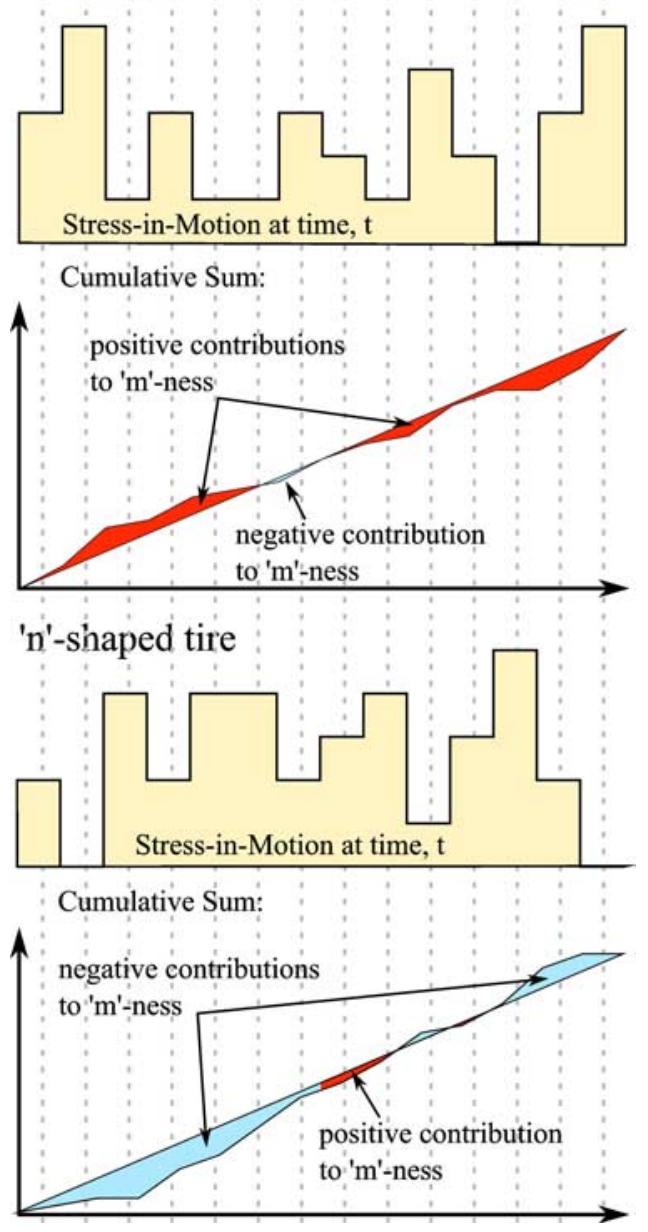

FIG. 9-Graphical representation of the basis of the " $m$ "-ness calculation.

respectively, (with the last two axles in a dual configuration), traveling at $70 \mathrm{~km} / \mathrm{h}$.

The primary purpose of this testing session was to record data from the accelerometers, shown as vibration sensors in Fig. 1. None

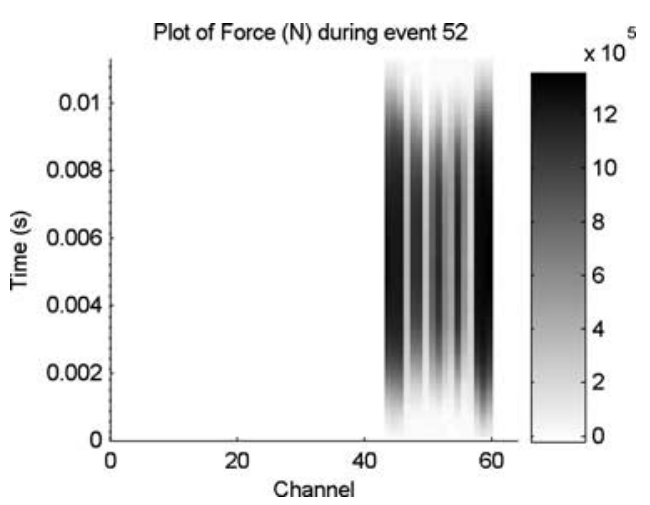

FIG. 10-SIM sensor results from a single tire with an " $m$ "-shaped stress distribution.

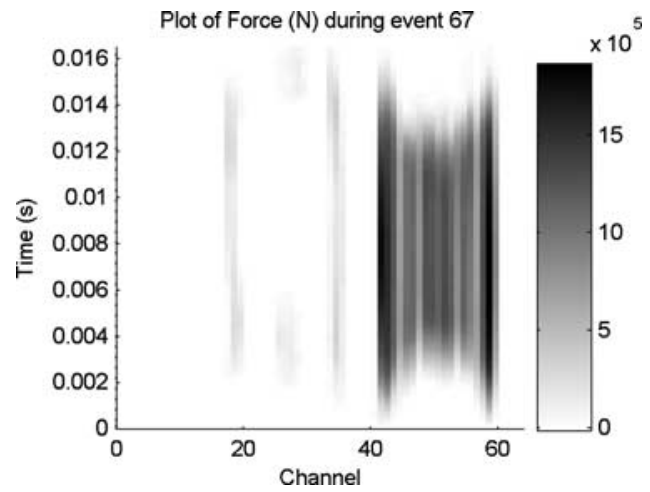

FIG. 11-SIM sensor results from a dual tire with " $m$ "-shaped stress distributions and one tire flat.

of the events recorded fully crossed the SIM sensor. Sufficient passes were made, however, that approximate stress distributions could be reconstructed from a number of truck passes and the known axle loads. It was these composite stress distributions that were used in the validation of the model.

A point in the wheel path thought to best represent the location of the acceleration sensor under the wheel was selected in the model and the model deflection at this point was recorded at a frequency of $255 \mathrm{~Hz}$ (to match the recording frequency of the accelerometers). The acceleration data were post-processed using a double integration method to give the deflection of the road surface during a vehicle pass and the two calculated deflections were compared. The results of this comparison for each vehicle are shown in Figs. 13 and 14.

The validation procedure will be repeated and the model improved using results of deformation sensors and various speeds.

Modeling Real Traffic - Real traffic data were taken from the recorded SIM sensor distributions from 2005. The model was used to predict the stresses and strains in the road due to the passing of single axles. It was assumed that the effect of any earlier axle would have entirely dissipated prior to the arrival of the modeled axle.

A number of axles were selected for modeling that fulfilled the following conditions:

- The axle was at least $1.8 \mathrm{~m}$ from any other axle (the WIM definition of a single axle).

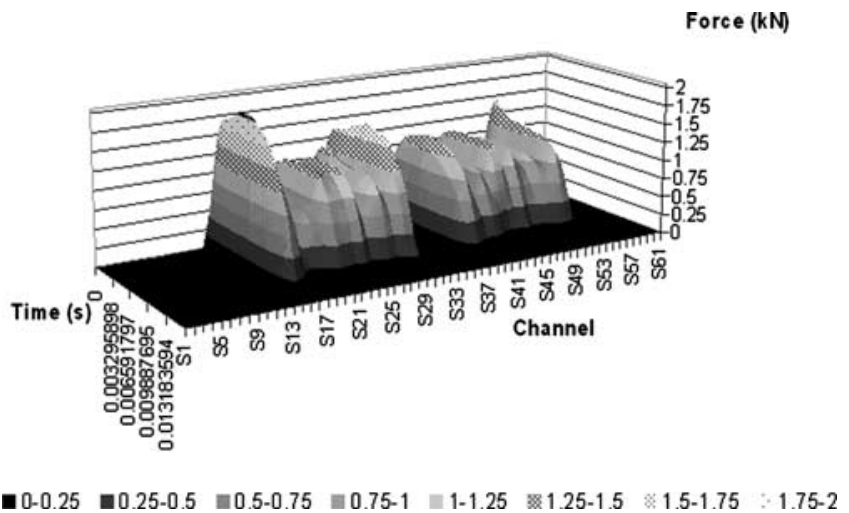

FIG. 12-SIM sensor results from a dual tire with " $m$ "-shaped stress distributions. Three-dimensional representation. 


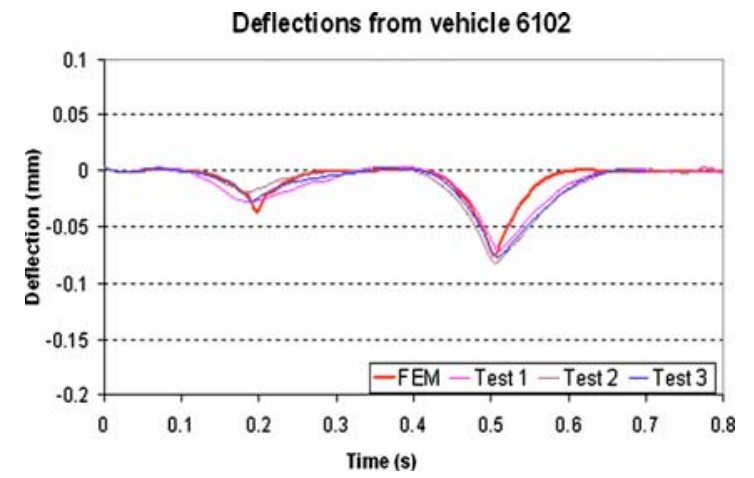

FIG. 13-Validation results for the FEM (Tests 1-3 reflects deflections calculated from acceleration data from three passes).

- The match between the WIM and SIM data for the axle was thought to be accurate.

Table 2 gives the information recorded by the FMS for some of the modeled axles. Where the wheels have two values of " $m$ "-ness and skew, the wheels were dual tires, the first value being for the innermost tire.

The plots discussed in the following sections are cross sections from the FEM results. The pavement under the wheel is shown with the hard shoulder to the left and the central median to the right. The wheel is traveling out of the page and its path is over the small, surface cells in the diagrams.

Results from Single Tires-Figures 15 and 16 show the results from the FEM for the two events with single tires. The effect of the different stress distribution shapes on the strain in the upper layer can be clearly seen. Comparing the figures in detail, we can

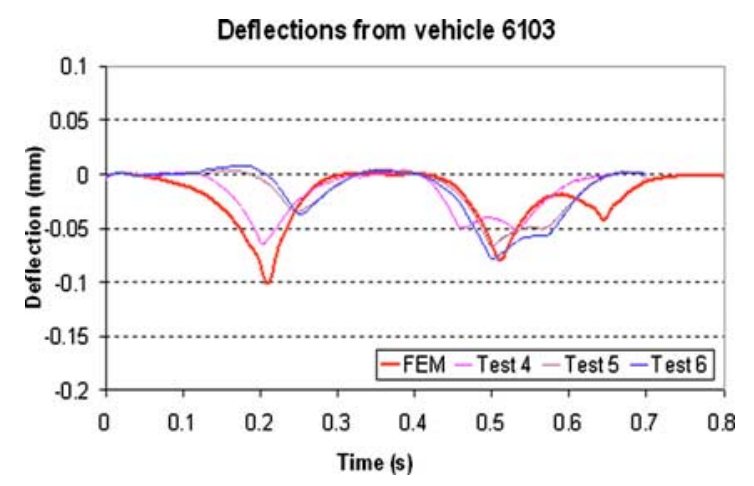

FIG. 14-Validation results from the FEM (Tests 4-6 reflects deflections calculated from acceleration data from three passes).

TABLE 2-Recorded properties of axles used with the FEM.

\begin{tabular}{lcccrr}
\hline ID & $\begin{array}{c}\text { Wheel Load } \\
(\mathrm{kN})\end{array}$ & $\begin{array}{c}\text { Speed } \\
(\mathrm{m} / \mathrm{s})\end{array}$ & Temperature $\left({ }^{\circ} \mathrm{C}\right)$ & “m”-ness & \multicolumn{1}{c}{ Skew } \\
\hline 52 & 50.5 & 25.0 & 27.4 & 0.9383 & -0.6324 \\
56 & 50.2 & 25.0 & 20.4 & 0.0745 & -0.5167 \\
58 & 50.6 & 25.0 & 24.3 & 0.0910 & 0.3832 \\
& & & & -0.1452 & 1.2560 \\
67 & 72.4 & 22.2 & 20.8 & 0.5764 & -0.5857 \\
& & & & 0.9123 & 0.6212 \\
70 & 83.9 & 21.7 & 24.5 & 0.7875 & -0.2304 \\
& & & & 0.3041 & 1.5445 \\
\hline
\end{tabular}

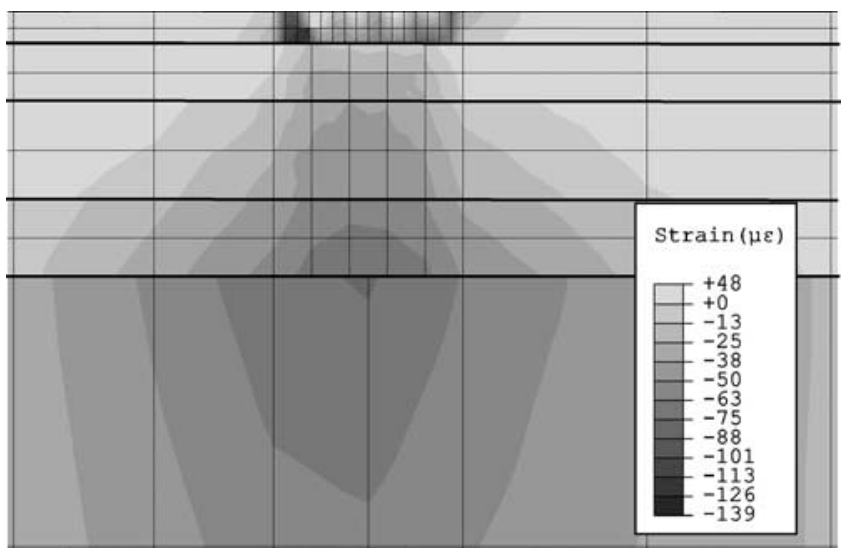

FIG. 15-Vertical compressive strain results from the FEM for run ID 52.

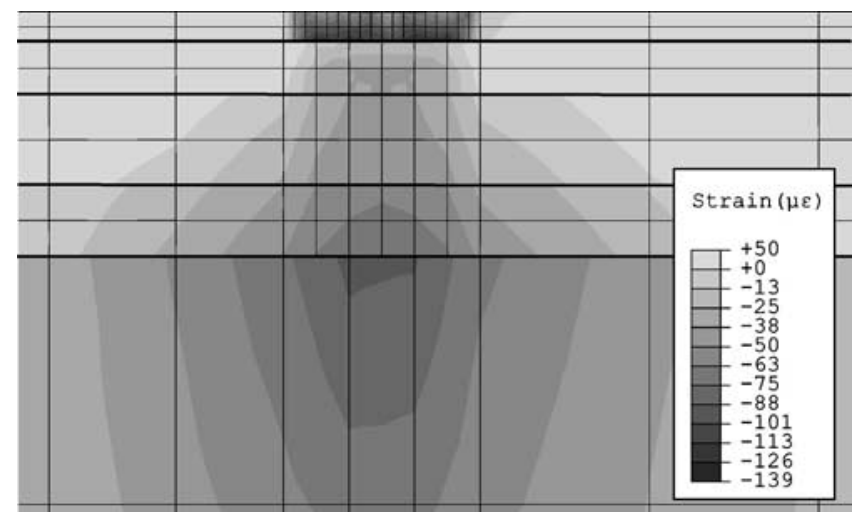

FIG. 16-Vertical compressive strain results from the FEM for run ID 56.

say that the "n"-shaped event 56 produces a very similar distribution to the "m"-shaped event 52 in all the layers except the surface layer. In the " $n$ "-shaped event more of the surface layer is strained close to the maximum strain of $139 \mu \epsilon$, whereas in the " $m$ "-shaped event a greater volume of the surface asphalt layer is strained, but to a lower level.

A comparison of the vertical tensile strains was also undertaken. The maximum vertical tensile strain from the " $n$ "-shaped distribution from the model was $27 \mu \epsilon$ compared to the "m"-shaped distribution which produced a maximum vertical tensile strain of $31 \mu \epsilon$. Both maxima occurred at the asphalt surface, just outside the wheel path.

Results from Double Tires-Figures 17 and 18 show the stress distributions from events $67^{4}$ and 70 , respectively. It can be seen that the maximum tensile and compressive stresses are both higher for event 67, despite event 70's higher loading. It is therefore clear that the presence of the flat or damaged tire on the vehicle in event 67 is significantly increasing the stresses in the pavement.

Figures 19 and 20 show vertical compressive stress distributions from events 58 and 70 . It can be seen that in event 70, with the higher wheel load, the strains in the subgrade and the base of the asphalt are dominant, and the strains near the surface of the pavement have not increased significantly by comparison. Conversely, in event 58, the strains at the base of the first asphalt layer (Layer 3)

${ }^{4}$ Note that event 67 is the event illustrated in Fig. 11, with the inner tire flat. 


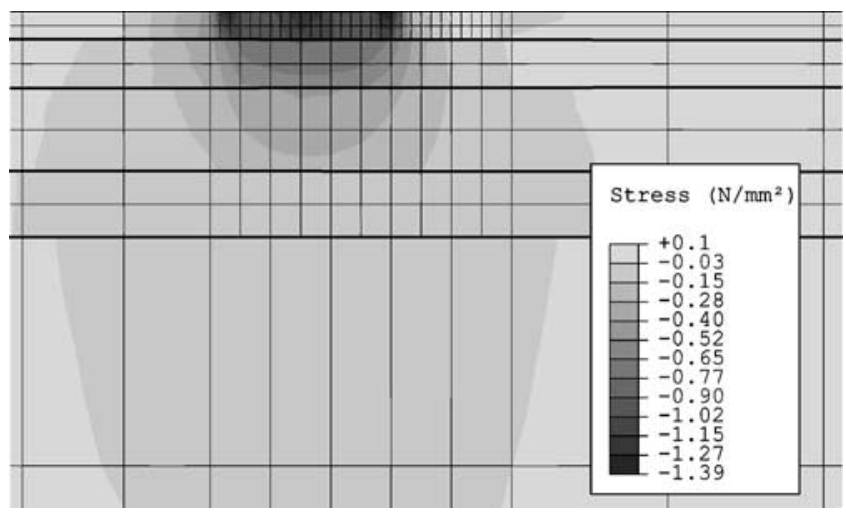

FIG. 17-Vertical stress distribution results from the FEM for run ID 67.

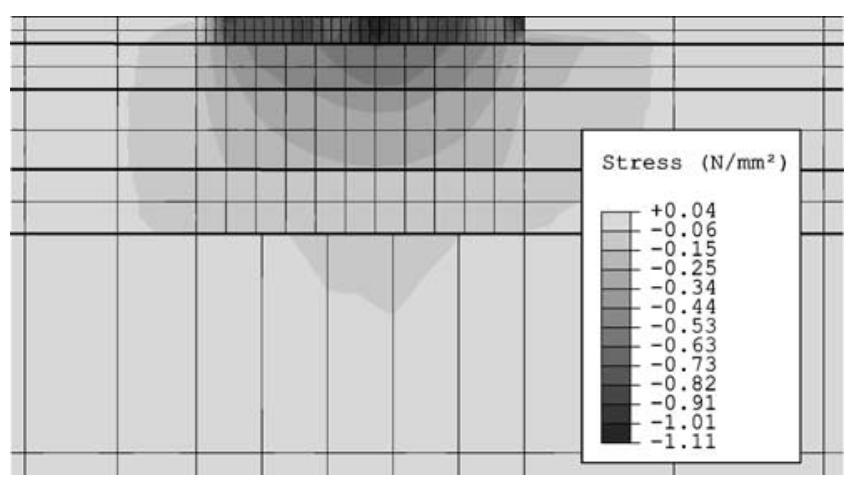

FIG. 18-Vertical stress distribution results from the FEM for run ID 70.

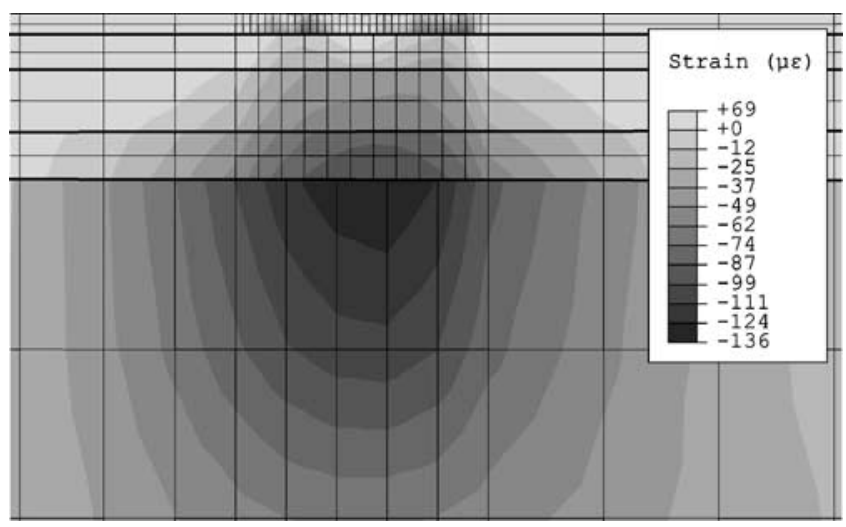

FIG. 19-Vertical compressive strain results from the FEM for run ID 58.

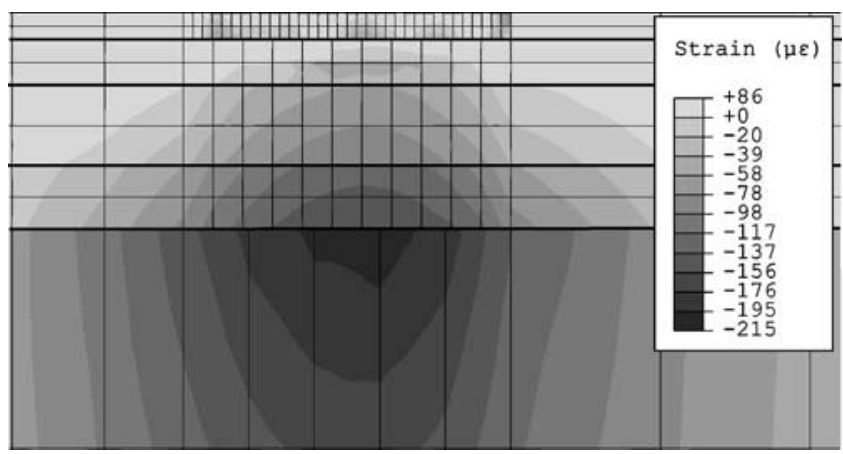

FIG. 20-Vertical compressive strain results from the FEM for run ID 70. are comparable in magnitude to those at the base of the pavement. These preliminary results suggest that the distribution shape is a more important factor in determining the peak pavement strain at low wheel loads. For very high wheel loads, the dominant factor is the wheel load itself.

\section{Conclusions}

The following conclusions have been drawn from this project:

- The existing weigh-in-motion station near Lenzburg was, and continues to be, a useful tool for the statistical assessment of traffic on the A1.

- The stress-in-motion sensor arrays have been successfully recording in situ data and are a useful addition to the WIM station, allowing for a greater understanding of the loading of the pavement.

- The data recorded by the SIM sensor can be effectively represented using the wheel load, "m"-ness, and skew parameters.

- The method for quantifying " $m$ "-ness proposed in the Distribution Shape section produces good agreement with the author's subjective shape classification.

- The finite element model described in the Finite Element Model section has been validated, reproducing the behavior of the road in response to dynamic loading to a reasonable level of accuracy.

- "m"-shaped, single tires produce higher tensile strains and lower compressive strains than " $n$ "-shaped tires at a given load.

- “m”-shaped, single tires strain a greater volume of asphalt than "n"-shaped tires.

- Vehicles which have improperly inflated, damaged, or flat tires have a much more significant effect on the pavement than those without.

- At very high wheel loads, the wheel load is a dominant factor in determining peak pavement strain; however, for all other wheels, the stress distribution has a significant effect.

- The shape of the tire's stress distribution has a very significant effect on the stresses and strains in the surface layer of asphalt at all wheel loads.

\section{Acknowledgments}

The authors would like to thank the Swiss Commission for Technology and Innovation (CTI/KTI), Swiss Federal Roads Authority (FEDRO/ ASTRA), Swiss Office for the Environment (FOEN/ BAFU), and Empa for their financial support, and Kistler Instruments AG, Switzerland and SIKA AG, Switzerland for supplying sensors and material, and Canton Aargau for their help throughout the project.

\section{References}

[1] De Beer, M., "Reconsideration of Tyre-Pavement Input Parameters for the Structural Design of Flexible Pavements," Proceedings 10th International Conference on Asphalt Pavements, Quebec, Canada, 2006.

[2] De Beer, M., Kannemeyer, L., and Fischer, C., "Towards Improved Mechanistic Design of Thin Asphalt Layer Surfaces 
Based on Actual Tyre/Pavement Contact Stress-in-Motion (SIM) Data in South Africa," 7th Conference on Asphalt Pavements for Southern Africa, 1999.

[3] De Beer, M., Fisher, C., and Jooste, F. J., "Determination of Pneumatic Tyre/Pavement Interface Contact Stresses Under Moving Loads and Some Effects on Pavements with Thin Asphalt Surfacing Layers," Proceedings, 8th International Conference on Asphalt Pavements, Seattle, WA, ICAP1997.

[4] Doupal, E. and Gysi, M., "Measurement of Dynamic Wheel Load Distributions," Proceedings of NATMEC - ICWIM-3 Congress, Orlando, FL, 2002.

[5] Ford, T. L. and Yap, P., “Truck Tire/Pavement Interface," Technical Papers Vol. II, XXIII FISITA Congress, Torino, Italy, 1990.

[6] Owende, M. O., Hartman, A. M., Ward, S. M., Gilchrist, M. D., and O’Mahony, M. J., "Minimizing Distress on Flexible Pavements Using Variable Tire Pressure," J. Transp. Eng., Vol. 127, 2001, pp. 254-262.

[7] Home page of the European cooperative projects, Eureka, http://www.Eureka.be/, Project Number E!2486, (2008).
[8] Kistler Catalogue, "Measurement of Wheel Load Distribution with MODULAS Sensor Type 9197," 53.609e-08.00. Kistler Instrumente AG Winterthur, CH 8408 Winterthur, Switzerland, 2005.

[9] Web site of Kistler Instrumente AG Switzerland: http// www.kistler.com/(2008).

[10] Arraigada, M., Partl, M. N., and Angelone, S. M., "Determination of Road Deflections from Traffic Induced Accelerations," International Journal of Road Materials and Pavement Design, JRMPD, Hermes Science Publications, Vol. 8/3, 2007, pp. 399-421.

[11] Massart, D. L., Smeyers-Verbeke, J., Capron, X., and Schlesier, K., "Visual Presentation of Data by Means of Box Plots," LC.GC Europe, Vol. 18, 2005, pp. 215-218.

[12] Poulikakos, L., Heutschi, K., Anderegg, P., Calderara, R., Doupal, E., Siegrist, R., and Partl, M. N., "Determination of the Environmental Footprint of Freight Vehicles," Proceedings, 4th International Conference on Weigh-in-Motion ICWIM4, Taipei, Taiwan, 2005.

[13] ABAQUS Documentation: Version 6.5; ABAQUS Inc., 2004. 